TRANSACTIONS OF THE

AMERICAN MATHEMATICAL SOCIETY

Volume 259, Number 2, June 1980

\title{
SOME CATEGORICAL EQUIVALENCES FOR $E$-UNITARY INVERSE SEMIGROUPS
}

BY

\author{
MARIO PETRICH
}

\begin{abstract}
The structure of $E$-unitary inverse semigroups has been described by McAlister and by Reilly and the author. The parameters in the first structure theorem may be made into a category, and the same holds for the parameters in the second structure theorem. We prove that each of these categories is equivalent to the category of $E$-unitary inverse semigroups and their homomorphisms. We also provide functors between the two first-mentioned categories which are naturally equivalent to the composition of the functors figuring in the categorical equivalence referred to above.
\end{abstract}

1. Introduction and summary. An inverse semigroup is $E$-unitary if it satisfies the implication $a e=e^{2}=e \Rightarrow a^{2}=a$. McAlister [1], [2] proved two remarkable theorems concerning these semigroups. (1) Every $E$-unitary inverse semigroup admits a faithful representation as a $P$-semigroup (which is reminiscent of a semidirect product of a semilattice and a group), and (2) every inverse semigroup is an idempotent separating homomorphic image of an $E$-unitary inverse semigroup.

The first-mentioned theorem of McAlister caused much research activity in the area of inverse semigroups. In particular, different proofs of this theorem were devised by Munn [4], Munn and Reilly [5] and Schein [7]. McAlister [2] also proved that morphisms of a McAlister triple $(Y, G ; X)$ are in a one-to-one correspondence with homomorphisms of the corresonding $E$-unitary inverse semigroup. A different, but related, structure theorem has been recently established by Reilly and the author [6]. While McAlister's construction of $E$-unitary inverse semigroups is based on groups acting on partially ordered sets by order automorphisms, the last-mentioned theorem is based on prehomomorphisms from groups into the inverse semigroups of isomorphisms among ideals of a semilattice.

In $\S 2$, we prove that there exists an equivalence of the category $\subseteq$ of $E$-unitary inverse semigroups and their homomorphisms and the category $\mathfrak{M}$ of McAlister triples and their morphisms. For the functor from $\subseteq$ to $\mathfrak{M}$, we use some elements of Schein's proof [7] of the nontrivial half of McAlister's theorem. We also use the correspondence of morphisms established by McAlister [2].

In $\S 3$, we perform a similar analysis but using unitary triples $(Y, G ; \psi)$ introduced in [6] instead of McAlister triples. Specifically, we prove that the category $\mathfrak{S}$

Received by the editors February 14, 1979 and, in revised form, September 17, 1979.

AMS (MOS) subject classifications (1970). Primary 20M10, 20M30, $18 \mathrm{~B} 10$.

Key words and phrases. E-unitary inverse semigroups, equivalence of categories, McAlister triples, unitary triples. 
is equivalent to the category $\mathfrak{U}$ of unitary triples and their morphisms. Here we use some relevant results established in [6].

$\S 4$ contains a functor from $\mathfrak{M}$ to $\mathfrak{U}$ which is naturally equivalent to the composition of the functors $\mathfrak{M} \rightarrow \mathfrak{S}$ and $\subseteq \rightarrow \mathfrak{U}$ which appear in the categorical equivalences in the preceding two sections.

Finally, in $\S 5$, we devise a functor from $\mathfrak{U}$ to $\mathfrak{M}$ which is naturally equivalent to the composition of the functors $\mathfrak{U} \rightarrow \subseteq$ and $\subseteq \rightarrow \mathfrak{M}$ which were used to establish the equivalence of these categories.

We refer to Mac Lane [3] for the background concerning category theory. We denote by $I_{\Subset}$ the identity functor on a category $\sqrt{ }$, and by $\iota_{X}$ the identity function on any set $X$. If $\varphi$ is a partial transformation on a set $X$, then $\delta \varphi$ and $\mathfrak{r} \varphi$ denote the domain and the range of $\varphi$, respectively.

2. E-unitary semigroups and McAlister triples. After introducing the necessary notation and terminology, we devise the two needed functors and the two natural equivalences which together will yield the desired categorical equivalence.

Note that if $Y$ is a subset of a partially ordered set $X$, then $Y$ is an ideal if $x<y$, $y \in Y$ implies $x \in Y ; Y$ is a subsemilattice of $X$ if any two elements of $Y$ have an infimum in $X$ which belongs to $Y$.

Definition 2.1. Let $X$ be a partially ordered set and $Y$ be an ideal and a subsemilattice of $X$. Let $G$ be a group acting on $X$ on the left by order automorphisms in such a way that $G Y=X$ and for every $g \in G$, we have $g Y \cap Y \neq \varnothing$. We call $(Y, G ; X)$ a McAlister triple (following [5]).

For a discussion of these triples, consult [1], [2], [5]. For morphisms, we have

Definition 2.2. Let $(Y, G ; X)$ and $\left(Y^{\prime}, G^{\prime} ; X^{\prime}\right)$ be McAlister triples. A pair $(\theta, \omega)$ is a morphism of $(Y, G ; X)$ into $\left(Y^{\prime}, G^{\prime} ; X^{\prime}\right)$ if

$(\alpha) \theta$ is an isotone mapping of $X$ into $X^{\prime}$,

( $\beta$ ) $\left.\theta\right|_{Y}$ is a semilattice homomorphism of $Y$ into $Y^{\prime}$,

$(\gamma) \omega$ is a homomorphism of $G$ into $G^{\prime}$,

$(\delta)(g \alpha) \theta=(g \omega)(\alpha \theta)$ for all $g \in G, \alpha \in X$.

Straightforward verification shows that these triples and their morphisms form a category. We may thus introduce

Notation 2.3. Let $\mathfrak{M}$ be the category consisting of McAlister triples and their morphisms. Let $\subseteq$ be the category of $E$-unitary inverse semigroups and their homomorphisms.

We now construct a functor from $\mathfrak{M}$ to $\mathfrak{S}$.

Definition 2.4. For each $(Y, G ; X) \in \mathrm{Ob} \mathfrak{M}$, let $P(Y, G ; X)$ be the set

$$
\left\{(\alpha, g) \in Y \times G \mid g^{-1} \alpha \in Y\right\}
$$

together with the multiplication

$$
(\alpha, g)(\beta, h)=(\alpha \wedge g \beta, g h) \text {. }
$$

Here $\gamma \wedge \delta$ denotes the infimum of the elements $\gamma, \delta \in X$ if it exists.

For each $(\theta, \omega) \in \operatorname{Hom} \mathfrak{M}$, say $(\theta, \omega):(Y, G, X) \rightarrow\left(Y^{\prime}, G^{\prime} ; X^{\prime}\right)$, let $P(\theta, \omega)$ be the mapping

$$
P(\theta, \omega):(\alpha, g) \rightarrow(\alpha \theta, g \omega) \quad((\alpha, g) \in P(Y, G ; X)) .
$$


LEMMA 2.5. With the notation just introduced, $P$ is a functor from $\mathfrak{M}$ to $\subseteq$ S.

Proof. Let $(Y, G ; X) \in \mathrm{Ob} \mathfrak{M}$. It is well known, see [1] and [2], and routine to verify, that $S=P(Y, G ; X)$ is an $E$-unitary inverse semigroup. Also for $(\theta, \omega) \in$ Hom $\mathfrak{R}$, it is straightforward to verify that $P(\theta, \omega)$ is a homomorphism of $P(Y, G ; X)$ into $P\left(Y^{\prime}, G^{\prime} ; X^{\prime}\right)$; this was also observed in [2, Theorem 6.1]. The remaining axioms for a functor are clearly satisfied.

We now devise a functor from $\subseteq$ to $\mathfrak{M}$. We denote by $\sigma$ and $E_{S}$ the least group congruence and the semilattice of idempotents of any inverse semigroup $S$.

Definition 2.6. Let $S \in \mathrm{Ob} \subseteq$ and denote $\mathcal{G}=S / \sigma, E=E_{S}$. For each $g \in \mathcal{G}$ and $e \in E$, let $g e=\left\{\left(s^{-1} e s, g(s \sigma)\right) \mid s \in S\right\}$. Further, let

$$
\mathcal{X}=\{g e \mid g \in \mathcal{G}, e \in E\}, \quad \mathcal{Y}=\{1 e \mid e \in E\}
$$

and let $\mathscr{X}$, and thus also $\mathcal{Y}$, be ordered by inclusion, and let $\mathcal{S}$ act on $\mathscr{X}$ by

$$
g(h e)=(g h) e \quad(g, h \in \mathcal{G}, e \in E) .
$$

Let $R(S)=(\mathcal{Y}, \mathcal{G} ; \mathcal{X})$ with this action.

Next let $\varphi \in$ Hom $\subseteq$, say $\varphi: S \rightarrow S^{\prime}$ is a homomorphism. Define $\omega$ and $\theta$ by

$$
(s \sigma) \omega=(s \varphi) \omega \sigma \quad(s \in S) ; \quad(g e) \theta=(g \omega)(e \varphi) \quad\left(g \in S / \sigma, e \in E_{S}\right),
$$

and let $R(\varphi)=(\theta, \omega)$.

LEMMA 2.7. With the notation just introduced, $R$ is a functor from $\subseteq$ to $\mathfrak{M}$.

Proof. Let $S \in \mathrm{Ob} \subseteq, \mathcal{G}=S / \sigma, E=E_{s}$. Let $g, h, k \in \mathcal{G}$ and $e, f \in E$. Assume that $g e \subseteq h f$. Since $(e, g) \in g e$, we get $(e, g) \in h f$ which implies that $(e, g)=$ $\left(s^{-1} f s, h(s \sigma)\right)$ for some $s \in S$. Hence $e=s^{-1} f s, g=h(s \sigma)$. Conversely, suppose that $e=s^{-1} f s$ and $g=s \sigma$ for some $s \in S$. Then for any $t \in S$, we have

$$
\left(t^{-1} e t, g(t \sigma)\right)=\left(t^{-1} s^{-1} e s t, h(s \sigma)(t \sigma)\right)=\left((s t)^{-1} e(s t), h(s t) \sigma\right) \in h f
$$

so that $g e \subseteq h f$. We have proved

$$
g e \subseteq h f \Leftrightarrow e=s^{-1} f s, \quad g=h(s \sigma) \quad \text { for some } s \in S .
$$

It follows that $g e \subseteq h f$ implies $(k g) e \subseteq(k h) f$, so by the definition of the action of $\mathcal{G}$ on $\mathcal{X}$, we obtain $k(g e) \subseteq k(h f)$. Conversely, if $k(g e) \subseteq k(h f)$, then applying $k^{-1}$, we obtain ge $\subseteq h f$. Consequently, $\mathcal{G}$ acts by order automorphisms on $\mathfrak{X}$ on the left.

Define a function $\psi$ by $\psi: e \rightarrow 1 e(e \in E)$. Assume that $e<f$ in $E$. Then for any $s \in S$, we get

$$
\left(s^{-1} e s, s \sigma\right)=\left(s^{-1} e f e s, s \sigma\right)=\left((e s)^{-1} f(e s),(e s) \sigma\right) \in 1 f
$$

and hence $1 e \subseteq 1 f$. Conversely, suppose that $1 e \subseteq 1 f$. Then $(e, 1) \in 1 e$ so $(e, 1)=$ $\left(s^{-1} f s, s \sigma\right)$ for some $s \in S$. It follows that $e=s^{-1} f s$ and $1=$ so. Hence $s \in E$ since $S$ is $E$-unitary and thus $e=s f \leqslant f$. Consequently, $\psi$ is an isomorphism of $E$ onto $\mathcal{Y}$. In particular, $\mathcal{Y}$ is a subsemilattice of $\mathcal{X}$.

In order to prove that $\mathcal{Y}$ is an ideal of $\mathcal{X}$, we assume that $g e \subseteq 1 f$. By (1), $e=s^{-1} f s$ and $g=s \sigma$ for some $s \in S$. Let $t=f s$ whence $e=t^{-1} t$ and $t \sigma=g$. We 
calculate

$$
\begin{aligned}
g e & =\left\{\left(u^{-1} e u, g(u \sigma)\right) \mid u \in S\right\}=\left\{\left(u^{-1} t^{-1} t u, g(u \sigma)\right) \mid u \in S\right\} \\
& =\left\{\left((t u)^{-1}\left(t t^{-1}\right)(t u),(t u) \sigma\right) \mid u \in S\right\} \subseteq 1\left(t t^{-1}\right) .
\end{aligned}
$$

Conversely, for $\left(v^{-1} t t^{-1} v, v \sigma\right) \in 1\left(t t^{-1}\right)$, letting $u=t^{-1} v$, we obtain

$$
\left(v^{-1} t t^{-1} v, v \sigma\right)=\left(\left(t^{-1} v\right)^{-1}\left(t^{-1} t\right)\left(t^{-1} v\right),\left(t t^{-1} v\right) \sigma\right)=\left(u^{-1} e u, g(u \sigma)\right) \in g e
$$

which implies that $g e=1\left(t t^{-1}\right)$. Consequently, $\mathcal{Y}$ is an ideal of $\mathcal{X}$.

By the very definitions, we have $\mathcal{G} \mathcal{Y}=\mathcal{X}$. Let $g \in \mathcal{G}$ and let $t \sigma=g$. Then

$$
\begin{aligned}
g\left(t^{-1} t\right) & =\left\{\left(s^{-1} t^{-1} t s, g(s \sigma)\right) \mid s \in S\right\} \\
& =\left\{\left((t s)^{-1}\left(t t^{-1}\right)(t s),(t s) \sigma\right) \mid s \in S\right\}=1\left(t t^{-1}\right),
\end{aligned}
$$

where the last equality is obtained as just above. Consequently, $g\left(t^{-1} t\right)=1\left(t t^{-1}\right)$ which proves $g \mathscr{Y} \cap \mathcal{O} \neq \varnothing$.

Therefore $(\mathcal{Y}, \mathcal{G} ; \mathfrak{X}) \in \mathrm{Ob} \mathfrak{M}$.

Now let $\varphi \in$ Hom $\subseteq$, say $\varphi: S \rightarrow S^{\prime}$ for $E$-unitary inverse semigroups $S$ and $S^{\prime}$. Let $s, t \in S$ be such that sot. Then $s e=t e$ for some $e \in E=E_{S}$, so that $(s \varphi)(e \varphi)$ $=(t \varphi)(e \varphi)$ which yields $s \varphi \sigma t \varphi$. This shows that $\omega$ defined by $(s \sigma) \omega=(s \varphi) \sigma$, ( $s \in S$ ) is single-valued. It is evident that $\omega$ is a homomorphism of $\mathcal{G}=S / \sigma$ into $\mathcal{G}^{\prime}=S^{\prime} / \sigma$.

Next let $g, h \in \mathcal{G}, e, f \in E$ and assume that $g e \subseteq h f$. By (1), we have $e=s^{-1} f s$ and $g=h(s \sigma)$ for some $s \in S$. Hence $e \varphi=(s \varphi)^{-1}(f \varphi)(s \varphi)$ and $g \omega=(h \omega)(s \sigma) \omega=$ $(h \omega)(s \varphi) \sigma$ which again by (1) means that $(g \omega)(e \varphi) \subseteq(h \omega)(f \varphi)$. Now considering the equality $g e=h f$, we conclude that the function $\theta$ defined by $(g e) \theta=(g \omega)(e \varphi)$, ( $g \in G, e \in E$ ) is single-valued. We have also shown that $\theta$ is isotone and maps $\mathcal{X}$ into $\mathcal{X}^{\prime}$ where $R(S)=(\mathcal{Y}, \mathcal{G} ; \mathcal{X})$ and $R\left(S^{\prime}\right)=\left(\mathcal{Y}^{\prime}, \mathcal{G}^{\prime} ; \mathcal{X}^{\prime}\right)$.

It is clear that $\mathcal{Y} \theta \subseteq \mathcal{Y}^{\prime}$. For any $e, f \in E$, we have

$$
(1 e \cap 1 f) \theta=(1(e f)) \theta=1((e f) \varphi)=1((e \varphi)(f \varphi))=1(e \varphi) \cap 1(f \varphi)
$$

since $\psi$ above is an isomorphism of $E_{S}$ onto $\mathcal{Y}$; analogously $E_{S^{\prime}}$ maps onto $\mathcal{Y}^{\prime}$. Hence $\left.\theta\right|_{9}$ is a homomorphism.

For any $g, h \in \mathcal{G}, e \in E$, we further obtain

$$
\begin{aligned}
{[g(h e)] \theta } & =[(g h) e] \theta=(g h) \omega(1 e) \theta=[(g \omega)(h \omega)](1 e) \theta \\
& =(g \omega)[(h \omega)(1 e) \theta]=(g \omega)(h e) \theta
\end{aligned}
$$

which concludes the proof that $R(\varphi)=(\theta, \omega)$ is a morphism of $R(S)$ into $R\left(S^{\prime}\right)$. Thus $R(\varphi) \in$ Hom $\mathfrak{M}$.

The remaining axioms for a functor can be verified without difficulty. Therefore $R$ is a functor from $\subseteq$ to $\mathfrak{M}$.

Lemma 2.8. For every $(Y, G ; X) \in \mathrm{Ob} \mathfrak{M}$, let $\tau(Y, G ; X)=(\theta, \omega)$ where

$$
\theta: g \alpha \rightarrow[(, g) \sigma][1(\alpha, 1)] \quad(g \in G, \alpha \in Y), \quad \omega: g \rightarrow(, g) \sigma \quad(g \in G),
$$

where the variables in the empty spaces are of no interest here. Then $\tau$ is a natural equivalence of the functors $I_{\mathfrak{M}}$ and $R P$. 
Proof. Let $(Y, G ; X) \in \mathrm{Ob} \mathfrak{M}$. Since in $S=P(Y, G ; X)$, we have $(\alpha, g) \sigma(\beta, h)$ if and only if $g=h$, it follows at once that $\omega$ is an isomorphism of $G$ onto $\mathcal{G}=S / \sigma$.

Next let $g, h \in G$ and $\alpha, \beta \in Y$. Assume that $g \alpha \leqslant h \beta$. Then $h^{-1} g \alpha<\beta$ and thus $h^{-1} g \alpha \in Y$, so $s=\left(h^{-1} g \alpha, h^{-1} g\right)$ is an element of $S$. Let $e=(\alpha, 1), f=$ $(\beta, 1)$, and calculate

$$
\begin{aligned}
s^{-1} f s & =\left(\alpha, g^{-1} h\right)(\beta, 1)\left(h^{-1} g \alpha, h^{-1} g\right)=\left(\alpha \wedge g^{-1} h \beta, g^{-1} h\right)\left(h^{-1} g \alpha, h^{-1} g\right) \\
& =\left(\alpha \wedge g^{-1} h \beta, 1\right)=(\alpha, 1)=e
\end{aligned}
$$

and $(, h) \sigma(s \sigma)=\left(, h h^{-1} g\right) \sigma=(, g) \sigma$. It now follows from (1) of Lemma 2.7 that

$$
[(, g) \sigma][1(\alpha, 1)] \subseteq[(, h) \sigma][1(\beta, 1)] \text {, }
$$

that is $(g \alpha) \theta \subseteq(h \beta) \theta$.

Conversely, assume that $(g \alpha) \theta \subseteq(h \beta) \theta$. Then by (1) of Lemma 2.7 , there exists $s \in S$ such that $(e,(, g) \sigma)=\left(s^{-1} f s,(, h) \sigma(s \sigma)\right)$ so that $e=s^{-1} f s,\left(, h^{-1} g\right) \sigma s$. It follows that $s=\left(\gamma, h^{-1} g\right)$ for some $\gamma \in Y$ such that $g^{-1} h \gamma \in Y$. Now

$$
\begin{aligned}
s^{-1} f s & =\left(g^{-1} h \gamma, g^{-1} h\right)(\beta, 1)\left(\gamma, h^{-1} g\right)=\left(g^{-1} h \gamma, g^{-1} h\right)\left(\beta \wedge \gamma, h^{-1} g\right) \\
& =\left(g^{-1} h(\beta \wedge \gamma), 1\right)=(\alpha, 1)=e
\end{aligned}
$$

which yields $g^{-1} h(\beta \wedge \gamma)=\alpha$. Hence $h^{-1} g \alpha=\beta \wedge \gamma<\beta$ and thus $g \alpha<h \beta$.

We have proved that $\theta$ is an isomorphism of partially ordered sets $X$ and

$$
\mathfrak{X}=\{[(, g) \sigma](1(\alpha, 1)) \mid g \in G, \alpha \in Y\} .
$$

It is clear that $\theta$ maps $Y$ onto $\mathcal{Y}=\{1(\alpha, 1) \mid \alpha \in Y\}$ where we set $[(, 1) \sigma][1(\alpha, 1)]$ $=1(\alpha, 1)$. For any $\alpha, \beta \in Y$, we further have

$$
\begin{aligned}
(\alpha \wedge \beta) \theta & =1(\alpha \wedge \beta, 1)=1[(\alpha, 1)(\beta, 1)] \\
& =1(\alpha, 1) \cap 1(\beta, 1)=\alpha \theta \cap \beta \theta,
\end{aligned}
$$

and hence $\left.\theta\right|_{Y}$ is a semilattice isomorphism of $Y$ onto $\mathcal{Y}$.

Let $g, h \in G, \alpha \in Y$; then

$$
\begin{aligned}
{[g(h \alpha)] \theta } & =[(g h) \alpha] \theta=[(, g h) \sigma][1(\alpha, 1)] \\
& =[(, g) \sigma]\{[(, h) \sigma][1(\alpha, 1)]\}=(g \omega)(h \alpha) \theta
\end{aligned}
$$

We finally note that $R P(Y, G ; X)=(\mathcal{Y}, \mathcal{G} ; \mathcal{X})$ as constructed above, and hence $(\theta, \omega)$ is an isomorphism of $(Y, G ; X)$ onto $R P(Y, G ; X)$.

It remains to establish the commutativity of the diagram

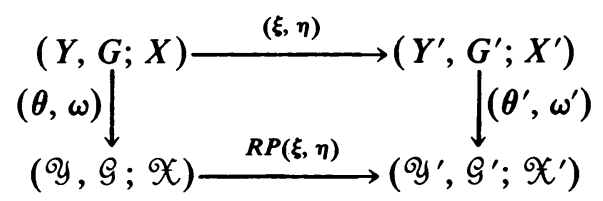

where $(\xi, \eta)$ is a morphism of $(Y, G ; X)$ into $\left(Y^{\prime}, G^{\prime} ; X^{\prime}\right)$ and the rest of the notation is obvious. Let $R P(\xi, \eta)=\left(\xi^{\prime}, \eta^{\prime}\right)$. For any $g \in G$, we have $g \eta \omega^{\prime}=$ $(, g \eta) \sigma=(, g) \sigma \eta^{\prime}=g \omega \eta^{\prime}$ and if also $\alpha \in Y$, then 


$$
\begin{aligned}
(g \alpha) \xi \theta^{\prime} & =[(g \eta)(\alpha \xi)] \theta^{\prime}=\left(g \eta \omega^{\prime}\right)[1(\alpha \xi, 1)]=(, g \eta) \sigma[1(\alpha, 1)] \xi^{\prime} \\
& =\{[(g) \sigma][1(\alpha, 1)]\} \xi^{\prime}=(, g \alpha) \theta \xi^{\prime} .
\end{aligned}
$$

LeMmA 2.9. For every $S \in \mathrm{Ob} \subseteq$, let $\chi=\chi(S)$ be defined by $\chi: s \rightarrow\left(1\left(s s^{-1}\right)\right.$, so), $(s \in S)$. Then $\chi$ is a natural equivalence of the functors $I_{\Im}$ and $P R$.

Proof. Let $S \in \mathrm{Ob} \subseteq$ and let $(\mathcal{Y}, \mathcal{G} ; \mathcal{X})=R(S), T=P(\mathcal{Y}, \mathcal{G} ; \mathcal{X}), \chi$ be as defined above. If $s \in S$, then

$$
\begin{aligned}
(s \sigma)^{-1}\left[1\left(s s^{-1}\right)\right] & =\left\{\left(t^{-1} s s^{-1} t,\left(s^{-1} t\right) \sigma\right) \mid t \in S\right\} \\
& =\left\{\left(\left(s^{-1} t\right)^{-1}\left(s s^{-1}\right),\left(s^{-1} t\right) \sigma\right) \mid t \in S\right\}=1\left(s^{-1} s\right) \in Y
\end{aligned}
$$

where the last equality is obtained similarly as in the proof of Lemma 2.7. Hence $\chi$ maps $S$ into $T$.

Next let $(1 e, g) \in T$. Then $g^{-1}(1 e) \in Y$ so that $g^{-1}(1 e)=1\left(t t^{-1}\right)$ for some $t \in S$ such that $e=t^{-1} t$ and $t \sigma=g$ as in the proof of Lemma 2.7. But then $t \chi=\left(1\left(t t^{-1}\right), s \sigma\right)=(e, g)$ and thus $\chi$ maps $S$ onto $T$.

Next assume that for $s, t \in S$, we have $\left(1\left(s s^{-1}\right), s \sigma\right)=\left(1\left(t t^{-1}\right), t \sigma\right)$. Then $1\left(s s^{-1}\right)$ $=1\left(t t^{-1}\right)$ and hence $s s^{-1}=t t^{-1}$ as we have seen in the proof of Lemma 2.7. Thus $s \Re t$ and $s \sigma t$ so that $s=t$ since $S$ is $E$-unitary. Consequently, $\chi$ is one-to-one.

In order to show that $\chi$ is a homomorphism, we first prove

$$
1\left(s s^{-1}\right) \cap(s \sigma)\left[1\left(t t^{-1}\right)\right]=1\left[(s t)(s t)^{-1}\right] .
$$

Let $(e, g) \in 1\left(s s^{-1}\right) \cap(s \sigma)\left[1\left(t t^{-1}\right)\right]$. Then

$$
(e, g)=\left(u^{-1} s s^{-1} u, u \sigma\right)=\left(v^{-1} t t^{-1} v,(s v) \sigma\right)
$$

so that $e=u^{-1} s s^{-1} u=v^{-1} t t^{-1} v$ and $g=u \sigma=(s v) \sigma$. We deduce

$$
\left(t t^{-1} v\right)^{-1}\left(t t^{-1} v\right)=v^{-1} t t^{-1} v=u^{-1} s s^{-1} u=\left(s^{-1} u\right)^{-1}\left(s^{-1} u\right)
$$

and hence $t t^{-1} v \subset s^{-1} v$. Further, $u \sigma=(s v) \sigma$ implies $\left(t t^{-1} v\right) \sigma=\left(s^{-1} u\right) \sigma$. It follows that $t t^{-1} v \mathcal{L} s^{-1} v$ and $t^{-1} t v \sigma s^{-1} u$ whence $t^{-1} t v=s^{-1} u$ since $S$ is $E$-unitary. Consequently,

$$
\begin{aligned}
e & =u^{-1} s\left(s^{-1} u\right)=u^{-1} s\left(t t^{-1} v\right)=u^{-1} s\left(t t^{-1}\right)\left(t t^{-1} v\right) \\
& =u^{-1} s\left(t t^{-1}\right) s^{-1} u=u^{-1}(s t)(s t)^{-1} u
\end{aligned}
$$

which together with $u \sigma=g$ yields $(e, g) \in 1\left[(s t)(s t)^{-1}\right]$.

Conversely, let $(e, g) \in 1\left[(s t)(s t)^{-1}\right]$, so that $(e, g)=\left(u^{-1} s t t^{-1} s^{-1} u^{-1}, u \sigma\right)$ for some $u \in S$, and thus $e=u^{-1} s t t^{-1} s^{-1} u, g=u \sigma$. It follows that

$$
e=\left(s^{-1} u\right)^{-1}\left(t t^{-1}\right)\left(s^{-1} u\right), \quad g=(s \sigma)\left(s^{-1} u\right) \sigma
$$

and hence $(e, g) \in(s \sigma)\left[1\left(t t^{-1}\right)\right]$. Since $(s t)(s t)^{-1}=s t t^{-1} s^{-1}<s s^{-1}$, by the proof of Lemma 2.7 , we have $1\left[(s t)(s t)^{-1}\right] \subseteq 1\left(s s^{-1}\right)$ which yields $(e, g) \in 1\left(s s^{-1}\right)$.

We have proved (2). It now follows that

$$
\begin{aligned}
(s \chi)(t \chi) & =\left(1\left(s s^{-1}\right), s \sigma\right)\left(1\left(t t^{-1}\right), t \sigma\right) \\
& =\left(1\left(s s^{-1}\right) \cap(s \sigma)\left[1\left(t t^{-1}\right)\right],(s \sigma)(t \sigma)\right) \\
& =\left(1\left[(s t)(s t)^{-1}\right],(s t) \sigma\right)=(s t) \chi
\end{aligned}
$$

and $\chi$ is indeed an isomorphism of $S$ onto $T$. 
Letting $\varphi$ be a homomorphism of $S$ into an $E$-unitary inverse semigroup $S^{\prime}$, we must show commutativity of the diagram

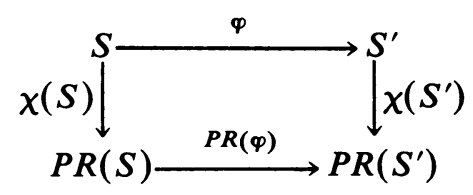

Indeed, for any $s \in S$, we have

$$
\begin{aligned}
s \varphi \chi\left(S^{\prime}\right) & =\left(1\left[(s \varphi)(s \varphi)^{-1}\right], s \varphi \sigma\right)=\left(1\left[\left(s s^{-1}\right) \varphi\right], s \varphi \sigma\right) \\
& =\left(1\left(s s^{-1}\right), s \sigma\right)[P R(\varphi)]=s[\chi(S)][P R(\varphi)] .
\end{aligned}
$$

Now collecting Lemmas 2.5, 2.7-2.9, we deduce

THeOREM 2.10. The quadruple $(P, R, \tau, \chi)$ is an equivalence of the categories $\mathfrak{M}$ and $\mathfrak{S}$.

3. E-unitary inverse semigroups and unitary triples. We first introduce the necessary notation and terminology, of which only "monoid prehomomorphism" and "unitary triple" are new. The functors and natural equivalences are then devised which make up the categorical equivalence we wanted to establish.

Definition 3.1. Let $S$ and $T$ be inverse semigroups. A mapping $\varphi: S \rightarrow T$ is a prehomomorphism if for all $a, b \in S$,

$(\alpha)(a \varphi)(b \varphi) \leqslant(a b) \varphi$,

( $\beta)(a \varphi)^{-1}=a^{-1} \varphi$.

If $S$ and $T$ are monoids and $\varphi$ also maps the identity of $S$ onto the identity of $T$, then $\varphi$ is a monoid prehomomorphism.

Notation 3.2. Let $Y$ be a semilattice, denote by $\Sigma(Y)$ the set of all isomorphisms among ideals of $Y$, written on the left and composed as such.

It is easy to verify that $\Sigma(Y)$ is closed under composition, and is thus an inverse semigroup. Its identity is $\iota_{Y}$, the identity transformation on $Y$. It is well known that for semigroups of one-to-one partial transformations, the canonical order coincides with the inclusion relation. Recall that for $\alpha \in \Sigma(Y)$, we denote by $\delta \alpha$ and $\mathrm{r} \alpha$ the domain and the range of $\alpha$, respectively.

Definition 3.3. A triple $(Y, G ; \psi)$ is a unitary triple if $Y$ is a semilattice, $G$ is a group, and $\psi$ is a monoid prehomomorphism of $G$ into $\Sigma(Y)$. We use the notation $\psi: g \rightarrow \psi_{g}$ but often write $g \alpha$ instead of $\psi_{g} \alpha$.

An ordered pair $(\theta, \omega)$ is a morphism of a unitary triple $(Y, G ; \psi)$ into another $\left(Y^{\prime}, G^{\prime} ; \psi^{\prime}\right)$ if $\theta: Y \rightarrow Y^{\prime}$ and $\omega: G \rightarrow G^{\prime}$ are homomorphisms such that for any $g \in G,(g \alpha) \theta=(g \omega)(\alpha \theta),\left(\alpha \in \delta \psi_{g}\right)$.

For a comprehensive discussion of unitary triples consult [6]. It is verified readily that unitary triples and their morphisms satisfy the axioms of a category. We may thus introduce

Notation 3.4. Let $\mathfrak{U}$ be the category of unitary triples and their morphisms.

In order to show that the categories $\mathfrak{U}$ and $\subseteq$ are equivalent, we first devise a functor from $\mathfrak{U}$ to $\mathfrak{S}$. 
Definition 3.5. For every $(Y, G ; \psi) \in \mathrm{Ob} \mathfrak{u}$, let $Q(Y, G ; \psi)$ be the set

$$
\left\{(\alpha, g) \in Y \times G \mid \alpha \in \mathfrak{r} \psi_{g}\right\}
$$

together with the multiplication

$$
(\alpha, g)(\beta, h)=\left(g\left(g^{-1} \alpha \wedge \beta\right), g h\right)
$$

where $\wedge$ denotes the operation in $Y$.

If $(\theta, \omega) \in \operatorname{Hom} \mathfrak{U}$, say $(\theta, \omega):(Y, G ; \psi) \rightarrow\left(Y^{\prime}, G^{\prime} ; \psi^{\prime}\right)$, let

$$
Q(\theta, \omega):(\alpha, g) \rightarrow(\alpha \theta, g \omega) \quad((\alpha, g) \in Q(Y, G ; \psi)) .
$$

LEMMA 3.6. With the notation just introduced, $Q$ is a functor from $\mathfrak{U}$ to $\mathfrak{S}$.

Proof. For $(Y, G ; \psi) \in \mathrm{Ob} \mathfrak{U}$, it was established in [6, Lemma 2.3] that $Q(Y, G ; \psi)$ is an $E$-unitary inverse semigroup, that is $Q(Y, G ; \psi) \in \mathrm{Ob}$ (5; a direct verification of this fact may be left to the reader.

Next let $(\theta, \omega) \in \operatorname{Hom} \mathfrak{U}$, say $(\theta, \omega):(Y, G ; \psi) \rightarrow\left(Y^{\prime}, G^{\prime} ; \psi^{\prime}\right)$. For any $(\alpha, g),(\beta, h) \in Q(Y, G ; \psi)$, letting $\varphi=Q(\theta, \omega)$, we obtain on the one hand,

$$
(\alpha, g) \varphi(\beta, h) \varphi=(\alpha \theta, g \omega)(\beta \theta, h \omega)=\left((g \omega)\left[(g \omega)^{-1}(\alpha \theta) \wedge \beta \theta\right],(g \omega)(h \omega)\right),
$$

and on the other hand,

$$
\begin{aligned}
{[(\alpha, g)(\beta, h)] \varphi } & =\left(g\left(g^{-1} \alpha \wedge \beta\right)\right) \varphi=\left(\left[g\left(g^{-1} \alpha \wedge \beta\right)\right] \theta,(g h) \omega\right) \\
& =\left((g \omega)\left[(g \omega)^{-1}(\alpha \theta) \wedge \beta \theta\right],(g \omega)(h \omega)\right)
\end{aligned}
$$

and $\varphi$ is a homomorphism. Thus $Q(\theta, \omega) \in$ Hom $\subseteq$.

The remaining axioms for a functor follow without difficulty.

For a functor from $\subseteq$ to $\mathfrak{U}$, we introduce

Definition 3.7. For every $S \in \mathrm{Ob} \subseteq$, let $T(S)=\left(E_{S}, S / \sigma ; \psi_{S}\right)$, where for all $g \in S / \sigma, \psi_{S}: g \rightarrow \psi_{g}$ is defined by $\psi_{g}\left(s^{-1} s\right)=s s^{-1}$ if $s \in S$, so $=g$.

If $\varphi: S \rightarrow S^{\prime}$ is a homomorphism of $E$-unitary inverse semigroups, let $T(\varphi)=$ $\left(\left.\varphi\right|_{E_{s}}, \omega\right)$, where $\omega$ is defined by $(s \sigma) \omega=s \varphi \omega,(s \in S)$.

The function $\psi_{S}$ is called the canonical prehomomorphism of $S$ in [6].

LEMMA 3.8. With the notation just introduced, $T$ is a functor from $\mathfrak{\subseteq}$ to $\mathfrak{U}$.

Proof. For any $S \in \mathrm{Ob} S$, it was established in [6, Lemma 3.1] that $\psi_{S}$ is a monoid prehomomorphism of $S / \sigma$ into $\Sigma\left(E_{S}\right)$. Hence $\left(E_{S}, S / \sigma ; \psi_{S}\right)$ is a unitary triple and thus $T(S) \in \mathrm{Ob} \mathfrak{U}$.

Next let $\varphi: S \rightarrow S^{\prime}$ be a homomorphism of $E$-unitary inverse semigroups, and let $\theta=\left.\varphi\right|_{E_{S}}$ and $\omega$ be as in Definition 3.7, so that $T(\varphi)=(\theta, \omega)$. Then $\theta$ is a homomorphism of $E_{S}$ into $E_{S^{\prime}}$, and $\omega$ is a homomorphism of $S / \sigma$ into $S^{\prime} / \sigma$ as can be easily verified. Let $g \in S / \sigma$ and $e \in \delta \psi_{g}$. Then $e=s^{-1} s$ and $s \sigma=g$ for some $s \in S$. Hence writing $g e=\psi_{g} e$, we get

$$
(g e) \theta=\left(s s^{-1}\right) \varphi=(s \varphi)(s \varphi)^{-1}=(g \omega)\left[(s \varphi)^{-1}(s \varphi)\right]=(g \omega)(e \theta)
$$

since $s \sigma=g$ implies $s \psi \sigma=s \sigma \omega=g \omega$. Consequently, $(\theta, \omega) \in$ Hom $\mathfrak{u}$.

The remaining axioms for a functor can be easily checked. 
Lemma 3.9. For every $(Y, G ; \psi) \in \mathrm{Ob} \mathfrak{U}$, define $\pi(Y, G ; \psi)=(\theta, \omega)$, where

$$
\theta: \alpha \rightarrow(\alpha, 1) \quad(\alpha \in Y) ; \quad \omega: g \rightarrow(, g) \sigma \quad(g \in G) .
$$

Then $\pi$ is a natural equivalence of the functors $I_{\mathfrak{u}}$ and $T Q$.

Proof. Let $(Y, G ; \psi) \in \mathrm{Ob} \mathfrak{U}$. Then $T Q(Y, G ; \psi)=(\mathcal{Y}, \mathcal{G} ; \Psi)$, where $\mathcal{Y}=$ $\{(\alpha, 1) \mid \alpha \in Y\}, \mathcal{G}=S / \sigma, S=Q(Y, G ; \psi)$, and

$$
\Psi_{(, g) \sigma}(\alpha, 1)=\left(\psi_{g} \alpha, 1\right) \quad\left(\alpha \in \delta \psi_{g}, g \in G\right)
$$

since

$$
\begin{aligned}
\Psi_{(, g) \sigma}(\alpha, 1) & =(\beta, 1) \\
& \Leftrightarrow(\alpha, 1)=s^{-1} s,(\beta, 1)=s s^{-1}, s \sigma=g \quad \text { for some } s \in S, \\
& \Leftrightarrow \alpha=\psi_{g^{-1}} \gamma, \beta=\gamma \text { if } s=(\gamma, g), \\
& \Leftrightarrow \beta=\psi_{g} \alpha .
\end{aligned}
$$

If $g \in G, \alpha \in \delta \psi_{g}$, then (1) yields $\Psi_{g \omega}(\alpha \theta)=\left(\psi_{g} \alpha\right) \theta$ which shows that $(\theta, \omega) \in$ Hom $\mathfrak{U}$. Since both $\theta$ and $\omega$ are isomorphisms, it follows that $(\theta, \omega)$ is an isomorphism of $(Y, G ; \psi)$ onto $(\mathcal{Y}, \mathcal{G} ; \Psi)$.

It remains to show commutativity of the diagram

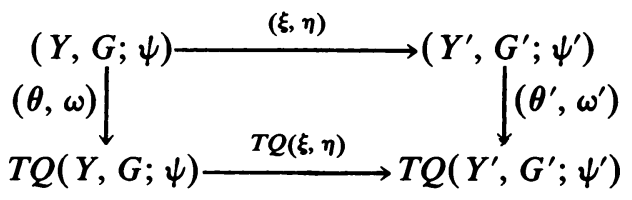

for a given $(\xi, \eta) \in$ Hom $\mathfrak{U}$. For any $g \in G$, letting $T Q(\xi, \eta)=\left(\xi^{\prime}, \eta^{\prime}\right)$, we have $g \eta \omega^{\prime}=(, g \eta) \sigma=(, g) \sigma \eta^{\prime}=g \theta \eta^{\prime}$ and for $\alpha \in Y, \alpha \xi \theta^{\prime}=(\alpha \xi, 1)=(\alpha, 1) \xi^{\prime}=\alpha \theta \xi^{\prime}$ which establishes commutativity of the diagram.

LemMA 3.10. For every $S \in \mathrm{Ob} \subseteq$, define $\zeta=\zeta(S)$ by $\zeta: s \rightarrow\left(s s^{-1}, s \sigma\right),(s \in S)$. Then $\zeta$ is a natural equivalence of the functors $I_{\Phi}$ and $Q T$.

Proof. Let $S$ and $\zeta$ be as in the statement of the lemma. We will use the canonical prehomomorphism $\psi_{S}: g \rightarrow \psi_{g}$, and will denote the multplication in $E_{S}$ by $\wedge$. Let $s \in S$ and $e<s^{-1} s$. Then $e \in \delta \psi_{s \sigma}$ and thus $e=t^{-1} t$, to $=s \sigma$ for some $t \in S$. Hence

$$
(s \sigma) e=(s \sigma)\left(t^{-1} t\right)=t t^{-1}=t\left(t^{-1} t\right) t^{-1}=t e t^{-1} .
$$

Now let $s, t \in S$ be arbitrary. Using (3), we obtain

$$
\begin{aligned}
(s t) \zeta & =\left((s t)(s t)^{-1},(s t) \sigma\right)=\left(s t t^{-1} s^{-1},(s t) \sigma\right) \\
& =\left(s\left[\left(s^{-1} s\right) \wedge\left(t t^{-1}\right)\right] s^{-1},(s t) \sigma\right) \\
& =\left((s \sigma)\left[(s \sigma)^{-1}\left(s s^{-1}\right) \wedge\left(t t^{-1}\right)\right],(s \sigma)(t \sigma)\right) \\
& =\left(s s^{-1}, s \sigma\right)\left(t t^{-1}, t \sigma\right)=(s \zeta)(t \zeta)
\end{aligned}
$$

and $\zeta$ is a homomorphism. For any $e \in E_{S}$ and $g \in S / \sigma$, we have

$$
\begin{aligned}
e \in \mathfrak{r} \psi_{g} & \Leftrightarrow e=s s^{-1} \text { and } s \sigma=g \text { for some } s \in S \\
& \Leftrightarrow s \zeta=(e, g) \text { for some } s \in S
\end{aligned}
$$


which implies that $\zeta$ maps $S$ onto $Q T(S)$. If $s \zeta=t \zeta$, then $s s^{-1}=t t^{-1}$ and $s \sigma=t \sigma$ so $s \mathcal{Q} \cap$ ot and thus $s=t$ since $S$ is $E$-unitary. Consequently, $\zeta$ is an isomorphism of $S$ onto $Q T(S)$.

If $\varphi: S \rightarrow S^{\prime}$ is a homomorphism of $E$-unitary inverse semigroups $S$ and $S^{\prime}$, then for any $s \in S$,

$$
s \varphi[\zeta(S)]=\left((s \varphi)(s \varphi)^{-1}, s \varphi \sigma\right)=\left(s s^{-1}, s \sigma\right)[Q T(\varphi)]=s[\zeta(S)][Q T(\varphi)]
$$

which proves commutativity of the diagram

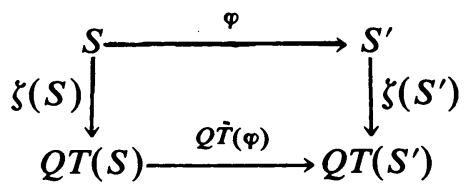

Collecting Lemmas 3.6, 3.8-3.10, we deduce

THEOREM 3.11. The quadruple $(Q, T, \pi, \zeta)$ is an equivalence of the categories $\mathfrak{U}$ and $\mathfrak{S}$.

4. From McAlister triples to unitary triples. Here we construct a functor from $\mathfrak{M}$ to $\mathfrak{U}$ and show that it is naturally equivalent to the composition $T P$.

Definition 4.1. For every $(Y, G ; X) \in \mathrm{Ob} \mathfrak{M}$, let $A(Y, G ; X)=\left(Y, G ; \psi_{X}\right)$ where $\psi_{X}: g \rightarrow \psi_{g}$ is given by $\psi_{g}: \alpha \rightarrow g \alpha$ if $\alpha, g \alpha \in Y$.

For every $(\theta, \omega) \in \operatorname{Hom} \mathfrak{M}$, say $(\theta, \omega):(Y, G ; X) \rightarrow\left(Y^{\prime}, G^{\prime} ; X^{\prime}\right)$, let $A(\theta, \omega)=$ $\left(\left.\theta\right|_{Y}, \omega\right)$.

LEMMA 4.2. With the notation just introduced, $A$ is a functor from $\mathfrak{M}$ to $\mathfrak{U}$.

Theorem 4.3. For every $(Y, G ; X) \in \mathrm{Ob} \mathfrak{M}$, define $\lambda(Y, G ; X)=(\theta, \omega)$ where

$$
\theta:(\alpha, 1) \rightarrow \alpha \quad(\alpha \in Y) ; \quad \omega:(, g) \sigma \rightarrow g \quad(g \in G) .
$$

Then $\lambda$ is a natural equivalence of the functors TP and $A$.

The proof is straightforward and is omitted.

5. From unitary triples to McAlister triples. We finally construct a functor from $\mathfrak{U}$ to $\mathfrak{M}$ and show that it is naturally equivalent to the composition $R Q$.

Definition 5.1. Let $(Y, G ; \psi) \in \mathrm{Ob} \mathfrak{U}$. For each $g \in G, \alpha \in Y$, define

$$
g \alpha=\left\{\left(\psi_{t}^{-1} \gamma, g t\right) \mid \gamma<\alpha, \gamma \in \mathfrak{r} \psi_{t}\right\}
$$

Let

$$
X_{\psi}=\{g \alpha \mid g \in G, \alpha \in Y\}, \quad Y_{\psi}=\{1 \alpha \mid \alpha \in Y\}
$$

be ordered by inclusion, with the action of $G$ on $X_{\psi}$ given by $g(h \alpha)=(g h) \alpha$, $(g, h \in G, \alpha \in Y)$. Define $B(Y, G ; \psi)=\left(Y_{\psi}, G ; X_{\psi}\right)$.

For a $\mathfrak{U}$-morphism $(\theta, \omega):(Y, G ; \psi) \rightarrow\left(Y^{\prime}, G^{\prime} ; \psi^{\prime}\right)$, define $B(\theta, \omega)=(\bar{\theta}, \omega)$ where $(g \alpha) \bar{\theta}=(g \omega)(\alpha \theta),(g \in G, \alpha \in Y)$.

LEMMA 5.2. With the notation just introduced, $B$ is a functor from $\mathfrak{U}$ to $\mathfrak{M}$.

The proof is omitted. 
TheOREM 5.3. For every $(Y, G ; \psi) \in \mathrm{Ob} \mathfrak{u}$, define $\rho(Y, G ; \psi)=(\xi, \eta)$ where

$$
\xi:[(, g) \sigma](\alpha, 1) \rightarrow g \alpha \quad(g \in G, \alpha \in Y) ; \quad \eta:(, g) \sigma \rightarrow g \quad(g \in G) .
$$

Then $\rho$ is a natural equivalence of the functors $R Q$ and $B$.

The proof is straightforward and is omitted.

\section{REFERENCES}

1. D. B. McAlister, Groups, semilattices and inverse semigroups, Trans. Amer. Math. Soc. 192 (1974), 227-244.

2. __ Groups, semilattices and inverse semigroups. II, Trans. Amer. Math. Soc. 196 (1974), 351-370.

3. S. Mac Lane, Categories for the working mathematician, Graduate Texts in Math., No. 5, Springer-Verlag, Berlin and New York, 1971.

4. W. D. Munn, A note on E-unitary inverse semigroups, Bull. London Math. Soc. 8 (1976), 71-76.

5. W. D. Munn and N. R. Reilly, E-unitary congruences on inverse semigroups, Glasgow Math. J. 17 (1976), 57-75.

6. M. Petrich and N. R. Reilly, A representation of E-unitary inverse semigroups, Quart. J. Math. (2) 30 (1979), 339-350.

7. B. M. Schein, A new proof for the McAlister P-theorem, Semigroup Forum 10 (1975), 185-188.

Institut de Mathímetiques, Universtté de MontPelliter, MontPellier, France

Department of Mathematics, Simon Fraser Universtty, Burnaby 2, British Columbla, Canada

Current address: Fachbereich 4/Mathematik, Universität Oldenberg, 2900 Oldenberg, Federal Republic of Germany 